\title{
VARIABILIDADE DE CARACTERÍSTICAS FÍSICAS E QUÍMICAS DE FRUTOS DE GERMOPLASMA DE BACURI DA REGIÃO MEIO-NORTE DO BRASIL ${ }^{1}$
}

\author{
VALDOMIRO AURÉLIO BARBOSA DE SOUZA²; EUGÊNIO CELSO EMÉRITO ARAÚJO ${ }^{33}$; \\ LÚCIO FLAVO LOPES VASCONCELOS 3 ; PAULO SARMANHO DA COSTA LIMA ${ }^{3}$
}

\begin{abstract}
RESUMO - Este trabalho teve como objetivo avaliar as características físicas e químicas de frutos de bacuri coletados de plantas matrizes de ocorrência na região Meio-Norte. As características analisadas foram: comprimento, largura e peso médio de fruto; peso médio de polpa; relação comprimento/largura, espessura de casca; percentagem de casca; percentagem de polpa; percentagem de sementes; número de sementes/fruto; número de secção partenocárpica/fruto; teor de sólidos solúveis totais; acidez total titulável e relação sólidos solúveis totais/acidez total titulável. Os frutos foram coletados de 26 plantas matrizes de bacuri mapeadas em nove locais de coleta no Piauí e Maranhão. Efetuou-se a avaliação das características físicas e químicas no Laboratório de Fisiologia Vegetal da Embrapa Meio-Norte, em Teresina-PI, utilizando-se de amostras de frutos de tamanho variável em função da disponibilidade de produção. Foi evidenciado o efeito significativo de local de coleta e de matrizes para todas as características estudadas, à exceção do número de secção partenocárpica/fruto para o qual não houve efeito de local de coleta. As características peso médio de fruto e peso médio de polpa; peso médio de fruto e largura de fruto; peso médio de polpa e largura de fruto; comprimento de fruto e espessura de casca; comprimento de fruto e percentagem de casca; espessura de casca e percentagem de casca, e peso médio de fruto e comprimento de fruto apresentaram altos valores de correlações fenotípicas $\left(r_{p} \geq 0,85\right)$. Estimativas de repetibilidade, variando de 0,50 (percentagem de polpa) a 0,98 (acidez total titulável), indicaram ampla variabilidade das características analisadas em relação ao efeito do ambiente permanente.
\end{abstract}

Termos para indexação: Platonia insignis, seleção, fruticultura tropical.

\section{VARIABILITY OF PHYSICAL AND CHEMICAL FRUIT CHARACTERISTICS OF BACURY GERMPLASM FROM MID-NORTH REGION OF BRAZIL}

\begin{abstract}
The objective of this study was to evaluate physical and chemical characteristics of bacury fruits collected from selected plants of occurrence in the Mid-North region. The following fruit characteristics were analysed: length e width; average weight; average polp weight; length to width ratio; peel thickness; percentage of peel; percentage of polp; percentage of seed; number of seeds/fruit; number of partenocarpic section/fruit; total soluble solids content; titratable acidity, and total soluble solids content to titratable acidity ratio. Fruits were collected from 26 selected plants mapped in nine locations in Piauí and Maranhão States. Physical and chemical fruit characterizatics were evaluated at the Plant Physiology Laboratory of Embrapa Meio-Norte, in Teresina, PI, Brazil. Fruit samples used varied in size according to the availability of fruit production from each selected plant. The variance analysis indicated significant effects for collect local and selected plants for all characteristics analysed, except for the number of partenocarpic section/fruit for which did not have local effect. Average fruit weight and average polp weight; average fruit weight and fruit width; average polp weight and fruit width; fruit length and peel thickness; fruit length and percentage of peel; peel thickness and percentage of peel; and average fruit weight and fruit length showed high values for phenotypic correlations $\left(\mathrm{r}_{\mathrm{p}}{ }^{3} 0.85\right)$. Repeatability estimates varied from 0.50 (\% POLP) to 0.98 (ATT) indicated large amount of variation for the characteristics analysed compared to permanent environment.
\end{abstract}

Index terms: Platonia insignis, selection, tropical fruit tree.

\section{INTRODUÇÃO}

O bacurizeiro (Platonia insignis Mart.), espécie frutífera da família Clusiaceae, é uma planta arbórea tipicamente tropical, cujas áreas de ocorrência abrangem os Estados do Pará, Maranhão, Piauí, Goiás e Mato Grosso, alcançando também o Paraguai (Ferreira et al., 1987; Macedo, 1995; Cavalcante, 1996). Os centros de origem e de diversidade da espécie estão localizados no Estado do Pará, onde é encontrada ampla variação de forma e tamanho de frutos, rendimento e qualidade de polpa, além de outras características de interesse econômico (Cavalcante, 1996).

Seu fruto pode ser utilizado tanto na forma in natura como para a agroindústria (Ferreira et al., 1987). A produção de bacuri é comercializada, principalmente, nas CEASAs e feiras livres de Belém-PA, São Luís, MA e Teresina-PI, e não tem sido suficiente para atender à demanda crescente do mercado

1 (Trabalho 236/2000). Recebido: 23/10/2000. Aceito para publicação: 01/11/2001. Trabalho desenvolvido com suporte financeiro do financeiro do Programa Avança Brasil.Programa Avança Brasil.

2 Eng. Agr., Ph.D., Pesquisador da Embrapa Meio-Norte, Cx. P. 01, CEP. 64.006-220, Teresina-PI. E-mail: valdo@cpamn.embrapa.br

3 Eng. Agr., M.Sc., Pesquisadores da Embrapa Meio-Norte. 
consumidor dessas capitais. Na forma de polpa congelada, a comercialização é feita, principalmente, nas grandes redes de supermercados dessas capitais a preços superiores aos de outras frutas tropicais como o cupuaçu, o cajá, a goiaba e a graviola, por exemplo. Portanto, a médio ou a longo prazos, essa espécie pode estabelecer-se como uma nova e excelente alternativa para os mercados interno e externo de frutas exóticas.

No entanto, apesar da sua importância social e do seu elevado potencial econômico, muito pouco tem sido feito para o conhecimento e uso dessa espécie, quer na área de coleta, conservação, caracterização e avaliação de germoplasma, quer na de melhoramento genético, visando ao desenvolvimento de cultivares ou de práticas adequadas de cultivo e manejo. Atualmente, o seu sistema de exploração, para o aproveitamento do fruto ou da madeira, é quase exclusivamente extrativista (Cavalcante, 1996; Moraes et al., 1994; Villachica et al., 1996).

$\mathrm{Na}$ área de germoplasma, o estudo de Guimarães et al. (1992) é um dos poucos encontrados na literatura. Esses autores coletaram e caracterizaram frutos de 15 matrizes de bacurizeiro de ocorrência na Ilha do Marajó-PA. Nas áreas de melhoramento genético, aproveitamento do fruto e técnicas de cultivo, esses estudos são ainda mais escassos. Na área de propagação, embora Carvalho et al. (1999) tenham desenvolvido estudos recentes sobre sistemas alternativos para a formação de mudas, muito ainda precisa ser feito para que o bacurizeiro possa ser cultivado de forma racional.

Na região Meio-Norte, em função dos desmatamentos, especialmente em áreas de cerrado, e do crescimento das áreas urbanas, acredita-se que boa parte da variabilidade genética existente nessa espécie já tenha sido perdida. Poucos esforços têm sido empreendidos pelas instituições de ensino e de pesquisa locais para resgatar e dar valor ao uso de germoplasma dessa preciosa fonte de alimentos e, assim, garantir a sua sustentabilidade para uso das gerações futuras.

Somente mais recentemente, a Embrapa Meio-Norte vem desenvolvendo esforços tanto na área de germoplasma como nas de propagação e técnicas de cultivo, visando a desenvolver tecnologias que permitam viabilizar a exploração econômica dessa frutífera na região.

Este trabalho teve por objetivos realizar a caracterização física e química de frutos de bacuri coletados de matrizes selecionadas em nove pontos de ocorrência da espécie na região Meio-Norte do Brasil, e estudar a repetibilidade e as correlações fenotípicas entre as características analisadas.

\section{MATERIAL E MÉTODOS}

As expedições de coleta foram realizadas no período de janeiro a março de 1998, período esse de maior concentração da produção do bacurizeiro na região. Foram selecionadas e cadastradas 71 matrizes em nove pontos de ocorrência da espécie nos Estados do Piauí e Maranhão. Contudo, em função da disponibilidade de produção por ocasião das expedições de coleta, foram incluídas neste trabalho apenas 26 matrizes $\left(\mathrm{M}_{1} \mathrm{PP}_{2}\right.$, $\mathrm{M}_{2} \mathrm{PP}_{2}, \mathrm{M}_{4} \mathrm{PP}_{2}, \mathrm{M}_{6} \mathrm{PP}_{2}, \mathrm{M}_{1} \mathrm{PP}_{4}, \mathrm{M}_{2} \mathrm{PP}_{5}, \mathrm{M}_{3} \mathrm{PP}_{5}, \mathrm{M}_{4} \mathrm{PP}_{5}, \mathrm{M}_{6} \mathrm{PP}_{5}$, $\mathrm{M}_{7} \mathrm{PP}_{5}, \mathrm{M}_{1} \mathrm{MP}_{1}, \mathrm{M}_{3} \mathrm{MP}_{1}, \mathrm{M}_{5} \mathrm{MP}_{1}, \mathrm{M}_{1} \mathrm{MP}_{3}, \mathrm{M}_{2} \mathrm{MP}_{3}, \mathrm{M}_{4} \mathrm{MP}_{3}$, $\mathrm{M}_{7} \mathrm{MP}_{3}, \mathrm{M}_{8} \mathrm{MP}_{3}, \mathrm{M}_{9} \mathrm{MP}_{3}, \mathrm{M}_{3} \mathrm{MP}_{6}, \mathrm{M}_{1} \mathrm{MP}_{7}, \mathrm{M}_{2} \mathrm{MP}_{8}, \mathrm{M}_{4} \mathrm{MP}_{8}$, $\mathrm{M}_{9} \mathrm{MP}_{8}, \mathrm{M}_{3} \mathrm{MP}_{9} \mathrm{e}_{4} \mathrm{MP}_{9}$ ). Na codificação das plantas matrizes, apresentada entre parênteses, $\mathrm{o} M$ numerado significa matriz e o não-numerado representa Maranhão; $\mathrm{o} P$ não numerado significa Piauí e o numerado local de coleta $\left(\mathrm{P}_{1}=\right.$ Caxias-MA, $\mathrm{P}_{2}=$ Palmeirais$\mathrm{PI}, \mathrm{P}_{3}=$ Matões-MA, $\mathrm{P}_{4}=$ Piracuruca-PI, $\mathrm{P}_{5}=$ Barras-PI, $\mathrm{P}_{6}=$ Aldeias Altas-MA, $\mathrm{P}_{7}=$ Codó-MA $\mathrm{P}_{8}=$ Brejo-MA e $\mathrm{P}_{9}=$ Santa QuitériaMA).

A caracterização física e química foi realizada no Laboratório de Fisiologia Vegetal da Embrapa Meio-Norte, em Teresina-PI. Chegados ao laboratório, os frutos coletados foram pesados individualmente, e as seguintes características foram analisadas: comprimento (CF) e largura ( $\mathrm{LF})$, em cm; relação comprimento/largura (CF/LF); peso médio de fruto (PMF) e peso médio de polpa (PMP), em gramas; espessura de casca (ECASC), em cm; percentagem de casca (\% CASC); percentagem de polpa (\% POLP); percentagem de sementes (\% SEM); número de sementes/fruto (NSEM/F); número de secção partenocárpica/ fruto (NSP/F); teor de sólidos solúveis totais (STT); acidez total titulável (ATT); e relação sólidos solúveis totais/acidez total titulável (STT/ATT). A relação CF/LF foi determinada dividindose as medidas de CF e LF dos frutos, tomadas com um paquímetro digital. As determinações do SST e da ATT foram feitas com base em amostras de $10 \mathrm{~g}$ de polpa diluída 1:10 (v:v). O STT foi determinado através da leitura em refratômetro de bolso, com compensação automática de temperatura, sendo os resultados expressos em \%. A ATT foi obtida por titulometria, usando $\mathrm{NaOH}$ a $0,1 \mathrm{~N}$ e fenolftaleína como indicador, sendo os resultados expressos em \% de ácido cítrico (Association..., 1975). A relação STT/ATT foi obtida dividindo-se o conteúdo de STT pelo de ATT. Foram utilizadas amostras de frutos de tamanho variável em função da disponibilidade de frutos maduros por ocasião das expedições de coleta, indo desde dois até 15 frutos/matriz.

Os dados foram analisados por meio da análise de variância, onde frutos por matriz foram utilizados como observações fenotípicas repetidas no mesmo indivíduo, resultando, assim, em estimativas da repetibilidade para cada característica analisada. Estimaram-se, também, as correlações fenotípicas entre todas as características estudadas. As análises dos dados foram realizadas utilizando-se os procedimentos PROC GLM e PROC CORR do Programa SAS (Littell et al., 1994) Na análise de variância, utilizou-se o teste $\operatorname{Scott-Knott~}(\mathrm{P}<0,05)$ para a comparação das médias entre pontos de coleta e entre matrizes, e o teste F para comparar as médias entre os Estados do Piauí e Maranhão.

\section{RESULTADOS E DISCUSSÃO}

A comparação da variabilidade fenotípica média do germoplasma coletado nos Estados do Piauí e Maranhão é apresentada na Tabela 1. Em média, as matrizes coletadas no Piauí apresentaram comprimento de fruto (CF), peso médio de fruto (PMF), peso médio de polpa (PMP), percentagem de polpa (\% POLP) e acidez total titulável (ATT) superiores aos das coletadas no Maranhão, enquanto o inverso ocorreu apenas para a relação sólidos solúveis totais/acidez total titulável (STT/ ATT). Para as demais características, os valores obtidos foram bastante similares entre os dois Estados.

A análise de variância indicou efeito significativo entre locais de coleta (Tabela 2 e 3 ) e entre matrizes (Tabela 4) para 
todas as características estudadas, à exceção do número de secção partenocárpica/fruto (NSP/F), que não apresentou efeito de local de coleta. Em média, as matrizes de Caxias, Santa Quitéria e Aldeias Altas, no Maranhão, e de Palmeirais e Barras, no Piauí, apresentaram as maiores médias para $\mathrm{CF}$, diferindo estatisticamente daquelas dos demais locais de coleta (Tabela 2). Em relação à largura de fruto (LF), sobressaíram-se as matrizes de Aldeias Altas e Caxias, no Maranhão, e Palmeirais, no Piauí. Por sua vez, as matrizes de Santa Quitéria e Caxias, no Maranhão, e Barras e Piracuruca, no Piauí, tiveram as maiores médias para a razão comprimento/largura de fruto $(\mathrm{CF} / \mathrm{LF})$. Em média, os valores da relação $\mathrm{CF} / \mathrm{LF}$ encontrados neste estudo foram superiores aos obtidos por Barbosa et al. (1979).

Quanto a PMF, as matrizes localizadas em Aldeias Altas, Santa Quitéria e Caxias, no Maranhão, e Palmeirais, no Piauí, tiveram as maiores médias e diferiram significativamente daquelas dos demais locais de coleta. As matrizes de Aldeias Altas, Santa Quitéria e Palmeirais também tiveram as maiores médias de PMP, enquanto as de Piracuruca, Caxias, Barras e Palmeirais foram as mais promissoras em termos de \% POLP, com valores médios entre 15,95 e 19,30\%. As matrizes de Santa Quitéria tiveram a menor média para esta característica (10,58\%), embora sem diferir daquelas de Brejo, Matões, Aldeias Altas e Codó, todos no Maranhão (Tabela 2). Teixeira (2000), analisando características físicas e químicas de frutos provenientes da CEASA-PI, encontrou médias para \% POLP um pouco superiores, com valores variando de 16,29 (frutos de coloração verde) a 18,35\% (frutos de coloração amarela).

As maiores médias para espessura de casca (ECASC) (Tabela 2) e percentagem de casca (\% CASC) (Tabela 3) foram obtidas pelas matrizes de Aldeias Altas e Santa Quitéria, sendo que, para \% CASC, não diferiram das matrizes de Palmeirais, Brejo, Piracuruca e Barras. A menor média de $\operatorname{ECASC}(0,80 \mathrm{~cm})$ foi obtida em Piracuruca. Em geral, o comportamento das matrizes em relação à percentagem de sementes (\% SEM) ocorreu de maneira inversa ao de \% CASC, o que concorda com resultados da literatura (Guimarães et al., 1992). As matrizes de Santa Quitéria, Aldeias Altas e Palmeirais apresentaram médias inferiores às das matrizes dos demais locais de coleta para esta característica. Em média, as matrizes de Palmeirais, Aldeias Altas e Brejo superaram as dos demais locais de coleta em termos de número de sementes/ fruto (NSEM/F), com médias de 3,82, 3,00 e 2,83 sementes/fruto, respectivamente. A média de todas as matrizes ficou em 2,44 sementes/fruto, concordando com dados da literatura (Cavalcante, 1996). Em relação a STT, a matriz de Codó foi a mais promissora, com 20,87\%. As matrizes de Santa Quitéria (16,22\%), Palmeirais $(15,83 \%)$ e Aldeias Altas $(15,10 \%)$ também apresentaram valores satisfatórios de STT (Tabela 3). Em média, as matrizes de Matões (9,54\%) tiveram o pior comportamento para essa característica, sem diferir, contudo, das matrizes de Caxias, Barras, Piracuruca e Brejo. Considerando todas as matrizes, a média de STT ficou em 14,21\% e não diferem muito daqueles encontrados na literatura (Santos et al., 1988; Nazaré, 2000; Teixeira, 2000).

Os frutos mais ácidos foram obtidos pelas matrizes de Caxias, Palmeirais e Matões, com valores médios de ATT de 2,02, 1,95 e 1,93\%, respectivamente. Contrariamente, as matrizes de Santa Quitéria obtiveram a melhor média para a relação STT/ ATT $(26,16)$. As matrizes Matões, Caxias, Palmeirais, Brejo e
Aldeias Altas tiveram as menores médias para essa relação. Nazaré (2000) e Santos et al. (1988) encontraram valores médios de ATT e relação STT/ATT de 1,6\% e 10,25 e 1,2\% e 12,0, respectivamente, concordando com os valores médios obtidos neste estudo.

$\mathrm{Na}$ Tabela 4, são apresentadas as médias da relação $\mathrm{CF} /$ FL, PMF, \% POLP, \% CASC, NSP/F e relação STT/ATT para as 26 matrizes estudadas. $\mathrm{O}$ formato de fruto, avaliado pela relação $\mathrm{CF} / \mathrm{LF}$, permitiu separar as diversas matrizes em dois grupos: um formado por matrizes de frutos comprido-ovalados (relação $\mathrm{CF}$ / LF entre 1,67 e 1,28) e outro por matrizes de frutos ovalados a redondo-achatados (relação CF/LF entre 1,25 e 0,82). Teixeira (2000) obteveram relação CF/LF variando de 1,11 a 1,19, enquanto Guimarães et al. (1992) relataram, para esta relação, valores, entre 0,86 e 1,30, indicando que a variabilidade para formato de fruto do germoplasma deste estudo é bastante expressiva.

As matrizes $\mathrm{M}_{1} \mathrm{MP}_{1}$ (Caxias) e $\mathrm{M}_{1} \mathrm{PP}_{2}$ (Palmeirais) foram significativamente superiores às demais matrizes em relação a PMF, com 697 e 656 g, respectivamente. Também se sobressaíram em PMF as matrizes $\mathrm{M}_{4} \mathrm{PP}_{5}$ (Barras), $\mathrm{M}_{3} \mathrm{MP}_{6}$ (Aldeias Altas) e $\mathrm{M}_{6} \mathrm{PP}_{2}$ (Palmeirais), com valores superiores a $460 \mathrm{~g}$, embora sem diferirem estatisticamente de sete outras matrizes. Guimarães et al. (1992) encontraram PMF variando de 127,1 a 669,7 g, porém, à exceção de duas matrizes, todas as demais apresentaram PMF inferior a $370 \mathrm{~g}$. As matrizes $\mathrm{M}_{2} \mathrm{MP}_{5}$ (Barras) e $\mathrm{M}_{4} \mathrm{MP}_{3}$ (Matões) tiveram as maiores médias para \% POLP, com 20,81 e 20,45\%, respectivamente, sem, contudo, diferirem estatisticamente das matrizes $\mathrm{M}_{1} \mathrm{MP}_{4}, \mathrm{M}_{8} \mathrm{MP}_{3}, \mathrm{M}_{5} \mathrm{MP}_{1}, \mathrm{M}_{1} \mathrm{MP}_{1}, \mathrm{M}_{2} \mathrm{MP}_{2}, \mathrm{M}_{7} \mathrm{MP}_{3}$, e $\mathrm{M}_{1} \mathrm{MP}_{2}$. As matrizes de piores performances para esta característica foram $\mathrm{M}_{2} \mathrm{MP}_{3}$ e $\mathrm{M}_{1} \mathrm{MP}_{3}$ ((Matões) e $\mathrm{M}_{8} \mathrm{MP}_{8}$ (Brejo), com apenas 4,87, 6,05 e 7,24\%, respectivamente. As médias de \% POLP encontradas na literatura, em geral, variam de $12-13 \%$ (Barbosa et al., 1979; Cavalcante, 1996; Nazaré, 2000) até 15,618,3\% (Santos et al., 1988; Teixeira, 2000), embora uma matriz com 30,6\% de polpa já tenha sido relatada (Guimarães et al., 1992).

Em relação à \% CASC, 23,08\% das matrizes tiveram valores médios entre 77,2 e $85,0 \%$, onde a matriz $\mathrm{M}_{6} \mathrm{PP}_{5}$, de bacuri sem sementes, atingiu o maior valor; $46,15 \%$ apresentaram valores médios entre $67,95 \%$ e $72,95 \%$, e $30,77 \%$ valores abaixo de $67,5 \%$, sendo que, neste grupo, a matriz $\mathrm{M}_{4} \mathrm{MP}_{3}$ teve o menor valor $(62,8 \%)$. As matrizes do primeiro grupo tiveram, em geral, baixo rendimento de polpa, contrastando com as do último grupo, onde ficou a maioria das matrizes promissoras em termos de rendimento de polpa. A média de todas matrizes, para esta característica, ficou em 70,81\%, o que está em concordância com a literatura, cujos valores médios encontrados variam entre 67 e 75\% (Barbosa et al., 1979; Santos et al., 1988; Cavalcante, 1996; Teixeira, 2000), embora valores bem mais baixos $(52,18 \%)$ e também mais elevados $(82,69 \%)$ tenham sido relatados para matrizes individuais (Guimarães et al., 1992).

Quanto ao NSP/F, cerca de 53,85\% das matrizes apresentaram valores médios satisfatórios para esta característica, com variação de 2,50 a 3,14. A matriz $\mathrm{M}_{6} \mathrm{PP}_{5}$ (sem sementes) apresentou a maior média absoluta para NSP/F $(4,88)$. Contudo, os frutos desse espécime são pequenos em demasia e não apresentam valor comercial. Outra característica negativa desse tipo de bacuri é que sua polpa é dura e quebradiça, sendo pouco apropriada para consumo ao natural e uso na agroindústria de polpa. 
TABELA 1 - Comparação da variabilidade para características físicas e químicas de frutos de bacurizeiro entre matrizes coletadas nos Estados do Piauí e Maranhão.

\begin{tabular}{|c|c|c|c|c|c|}
\hline \multirow{2}{*}{$\begin{array}{c}\text { C a c a racterísticas } \\
\text { avaliadas }\end{array}$} & \multirow{2}{*}{$\begin{array}{c}\text { U n idade } \\
\text { de m edida }\end{array}$} & \multicolumn{2}{|c|}{ E s ta do } & \multirow{2}{*}{$M$ éd ia } & \multirow{2}{*}{$\begin{array}{l}\mathrm{C} \cdot \mathrm{V} . \\
(\%)\end{array}$} \\
\hline & & P ia u í & M a ran hão & & \\
\hline $\mathrm{C} \mathrm{F} * *$ & $\mathrm{c} \mathrm{m}$ & 10,11 & 9,33 & 9,72 & 10,32 \\
\hline $\mathrm{R}$ e $1 \mathrm{a}$ çã o $\mathrm{C} \mathrm{F} / \mathrm{L} \mathrm{F}$ N & & 1,27 & 1,19 & 1,23 & 8,07 \\
\hline $\mathrm{P} \mathrm{M} \mathrm{F} * *$ & g & 351,26 & 300,58 & 325,92 & 26,18 \\
\hline $\mathrm{E} C \mathrm{~A} \mathrm{SC}{ }^{\mathrm{NS}}$ & $\mathrm{cm}$ & 1,25 & 1,23 & 1,24 & 11,10 \\
\hline$\% \quad \mathrm{P} \mathrm{O} \mathrm{L} \mathrm{P} \mathrm{*}$ & & 15,95 & 13,79 & 14,87 & 21,69 \\
\hline$\% \mathrm{C} \mathrm{A} \mathrm{S} \mathrm{C}^{\mathrm{NS}}$ & & 70,37 & 71,09 & 70,73 & 4,53 \\
\hline$\% \quad \mathrm{~S} \mathrm{E} \mathrm{M} \mathrm{N} \mathrm{S}$ & & 13,68 & 15,13 & 14,40 & 23,74 \\
\hline $\mathrm{NSEM} / \mathrm{F}^{\mathrm{NS}}$ & $\mathrm{u} \mathrm{n}$ & 2,60 & 2,35 & 2,47 & 37,74 \\
\hline $\mathrm{A} \mathrm{T} \mathrm{T} * *$ & $\% \quad \mathrm{~A} \mathrm{c}^{2}$ & 1,32 & 1,15 & 1,24 & 17,54 \\
\hline $\mathrm{R}$ e la çã o $\mathrm{S} \mathrm{S} \mathrm{T} \mathrm{/} \mathrm{A} \mathrm{T} \mathrm{T} \mathrm{**}$ & & 11,01 & 12,49 & 11,75 & 52,47 \\
\hline
\end{tabular}

${ }^{1} \mathrm{CF}=$ Comprimento de fruto; $\mathrm{LF}=$ Largura de fruto; PMF $=$ Peso médio de fruto; $\mathrm{PMP}=$ Peso médio de polpa; ECASC $=$ Espessura de casca; $\%$ POLP $=$ Percentagem de polpa; $\%$ CASC $=$ Percentagem de casca; $\%$ SEM $=$ Percentagem de sementes; NSEM/F $=$ Número de sementes/fruto; NSP $/ F=$ Número de seç̧ão partenocárpica/fruto; STT $=$ Teor de sólidos solúveis totais; ATT = Acidez total titulável.

${ }^{2}$ Acido cítrico.

* Significatico a 0,05 , pelo teste F.

** Significatico a 0,01 .

NS - Não significativo.

TABELA 2 - Características físicas e químicas de frutos de plantas matrizes de bacurizeiro por ponto de coleta.

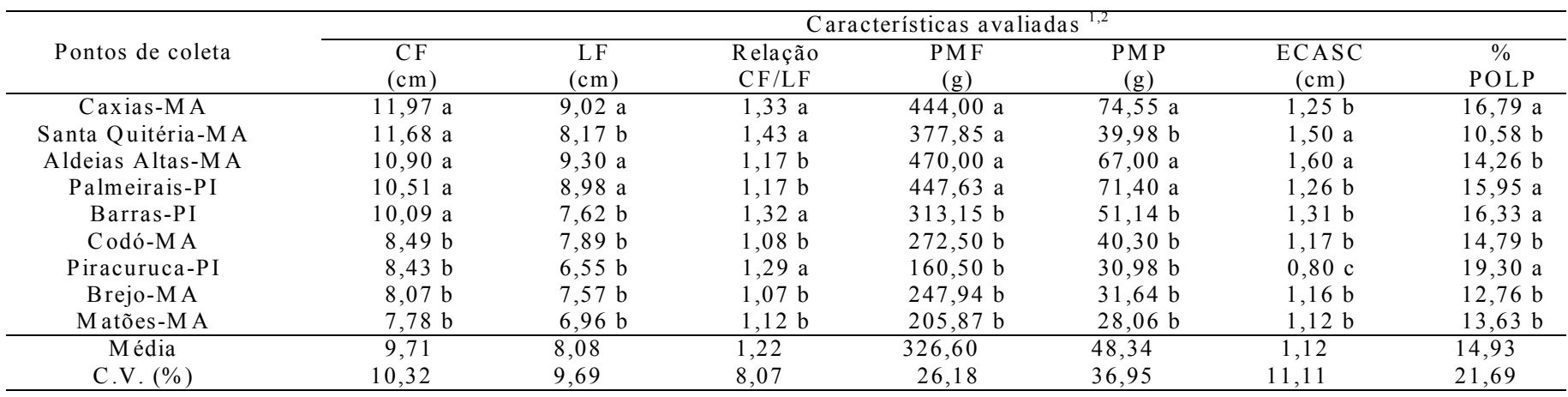

${ }^{1}$ Médias seguidas da mesma letra, na coluna, não diferem estatisticamente, pelo teste Scott-Knott, a $5 \%$.

${ }^{2} \mathrm{CF}=$ Comprimento de fruto; $\mathrm{LF}=$ Largura de fruto; $\mathrm{PMF}=$ Peso médio de fruto; $\mathrm{PMP}=$ Peso médio de polpa; ECASC $=$ Espessura de casca; $\%$ POLP $=$ Percentagem de polpa.

TABELA 3 - Características físicas e químicas de frutos de plantas matrizes de bacurizeiro por ponto de coleta.

\begin{tabular}{|c|c|c|c|c|c|c|c|}
\hline \multirow{3}{*}{ Pontos de coleta } & \multicolumn{7}{|c|}{ Características avaliadas ${ }^{1,2}$} \\
\hline & $\%$ & $\%$ & $\mathrm{NSEM} / \mathrm{F}$ & $\mathrm{NSP} / \mathrm{F}$ & STT & ATT & Relação \\
\hline & CASC & SEM & & & $(\%)$ & $\left(\% \mathrm{AC}^{3}\right)$ & STT/ATT \\
\hline Aldeias Altas-MA & $77,72 \mathrm{a}$ & $11,70 \mathrm{~b}$ & $3,00 \mathrm{a}$ & $2,00 \mathrm{a}$ & $15,10 \mathrm{~b}$ & $1,33 \mathrm{~b}$ & $11,35 \mathrm{c}$ \\
\hline Santa Quitéria-MA & $73,24 \mathrm{a}$ & $10,34 \mathrm{~b}$ & $2,35 \mathrm{~b}$ & $2,90 \mathrm{a}$ & $16,22 \mathrm{~b}$ & $0,62 \mathrm{~b}$ & $26,16 \mathrm{a}$ \\
\hline Palmeirais-PI & $71,10 \mathrm{a}$ & $12,11 \mathrm{~b}$ & $3,82 \mathrm{a}$ & $2,09 \mathrm{a}$ & $15,83 \mathrm{~b}$ & $1,95 \mathrm{a}$ & $8,12 \mathrm{c}$ \\
\hline Brejo-MA & $70,64 \mathrm{a}$ & $15,11 \mathrm{a}$ & $2,83 \mathrm{a}$ & $2,39 a$ & $10,95 \mathrm{c}$ & $1,04 \mathrm{~b}$ & $10,53 \mathrm{c}$ \\
\hline Piracuruca-PI & $69,95 \mathrm{a}$ & $16,42 \mathrm{a}$ & $1,50 \mathrm{~b}$ & $3,00 \mathrm{a}$ & $12,15 \mathrm{c}$ & -- & -- \\
\hline Barras-PI & $69,84 \mathrm{a}$ & $17,41 \mathrm{a}$ & $1,91 \mathrm{~b}$ & $2,68 \mathrm{a}$ & $13,51 \mathrm{c}$ & $0,82 \mathrm{~b}$ & $16,48 \mathrm{~b}$ \\
\hline Codó-MA & $68,78 \mathrm{~b}$ & $16,43 \mathrm{a}$ & $2,10 b$ & $2,50 \mathrm{a}$ & $20,87 \mathrm{a}$ & $1,27 \mathrm{~b}$ & $16,43 \mathrm{~b}$ \\
\hline Caxias-MA & $65,93 \mathrm{~b}$ & $18,12 \mathrm{a}$ & $2,50 \mathrm{~b}$ & $2,83 \mathrm{a}$ & $13,70 \mathrm{c}$ & $2,02 \mathrm{a}$ & $6,78 \mathrm{c}$ \\
\hline Matões-MA & $65,40 \mathrm{~b}$ & $15,34 \mathrm{a}$ & $1,96 \mathrm{~b}$ & $2,63 \mathrm{a}$ & $9,54 \mathrm{c}$ & $1,93 \mathrm{a}$ & $4,94 \mathrm{c}$ \\
\hline Média & 70,29 & 14,78 & 2,44 & 2,56 & 14,21 & 1,37 & 12,60 \\
\hline C.V. $(\%)$ & 4,53 & 23,74 & 37,74 & 42,14 & 16,87 & 23,28 & 52,47 \\
\hline
\end{tabular}

${ }^{1}$ Médias seguidas da mesma letra, na coluna, não diferem estatisticamente, pelo teste Scott-Knott, a 5\%.

$2 \% \mathrm{CASC}=$ Percentagem de casca; \% SEM = \% de sementes; NSEM/F = Número de sementes/fruto; NSP/F = Número de secção partenocárpica/fruto; STT

= Teor se sólidos solúveis totais; ATT = Acidez total titulável.

${ }^{3}$ Ácido cítrico. 
TABELA 4 - Relação comprimento/largura de fruto (CF/LF), peso médio de fruto (PMF), percentagem de polpa (\% POLP), percentagem de casca (\% CASC), número de secção partenocárpica/fruto e relação sólidos solúveis totais/acidez total titulável (STT/ATT) de 26 plantas matrizes de bacurizeiro coletadas no Meio-Norte do Brasil.

\begin{tabular}{|c|c|c|c|c|c|c|}
\hline M atriz $^{1,2}$ & $\begin{array}{c}\text { Relação } \\
\text { CF/LF }\end{array}$ & $\begin{array}{c}\text { PMF } \\
(\mathrm{g})\end{array}$ & $\%$ POLP & $\%$ CASC & $\mathrm{NSP} / \mathrm{F}$ & $\begin{array}{c}\text { Relação } \\
\text { STT/ATT } \\
\end{array}$ \\
\hline М 4P P 5 & $1,60 \mathrm{a}$ & $487,57 \mathrm{~b}$ & $13,25 \mathrm{~b}$ & 79,12 a & $3,14 \mathrm{a}$ & $22,12 \mathrm{c}$ \\
\hline M 4 M P 9 & $1,60 \mathrm{a}$ & $403,30 \mathrm{~b}$ & $9,38 \mathrm{c}$ & $77,20 \mathrm{a}$ & $2,70 \mathrm{a}$ & $52,62 \mathrm{~b}$ \\
\hline M 7 M P 3 & $1,51 \mathrm{a}$ & $135,00 \mathrm{~d}$ & $17,04 \mathrm{a}$ & $67,41 \mathrm{c}$ & $3,00 \mathrm{a}$ & - \\
\hline М 6 РP $5 *$ & $1,46 \mathrm{a}$ & $139,71 \mathrm{~d}$ & $14,46 \mathrm{~b}$ & $85,00 \mathrm{a}$ & $4,88 \mathrm{a}$ & $15,07 \mathrm{c}$ \\
\hline M 1 M P 1 & $1,40 \mathrm{a}$ & $697,00 \mathrm{a}$ & $17,74 \mathrm{a}$ & $71,56 \mathrm{~b}$ & $2,50 \mathrm{a}$ & $8,13 \mathrm{~d}$ \\
\hline M 9M P 8 & $1,40 \mathrm{a}$ & $167,33 \mathrm{~d}$ & $7,24 \mathrm{~d}$ & $71,43 \mathrm{~b}$ & $2,67 \mathrm{a}$ & $4,76 \mathrm{~d}$ \\
\hline M 3 PP 5 & $1,39 \mathrm{a}$ & $249,71 \mathrm{c}$ & $14,04 \mathrm{~b}$ & $69,72 \mathrm{~b}$ & $2,00 \mathrm{~b}$ & $18,14 \mathrm{c}$ \\
\hline M 3 M P 1 & $1,34 \mathrm{a}$ & $266,00 \mathrm{c}$ & $14,29 \mathrm{~b}$ & $71,05 \mathrm{~b}$ & $3,00 \mathrm{~b}$ & $5,83 \mathrm{~d}$ \\
\hline M 2 P P 2 & $1,32 \mathrm{a}$ & $379,41 \mathrm{~b}$ & $17,65 \mathrm{a}$ & $66,58 \mathrm{c}$ & $2,59 \mathrm{a}$ & $6,07 \mathrm{~d}$ \\
\hline M 7 P P 5 & $1,28 \mathrm{a}$ & $348,17 \mathrm{~b}$ & $13,88 \mathrm{~b}$ & $72,95 \mathrm{~b}$ & $3,00 \mathrm{~b}$ & $29,13 \mathrm{c}$ \\
\hline M 3 M P 9 & $1,25 \mathrm{~b}$ & $352,40 \mathrm{~b}$ & $11,78 \mathrm{c}$ & $78,25 \mathrm{a}$ & $3,10 \mathrm{a}$ & $16,16 \mathrm{c}$ \\
\hline M 1 PP 4 & $1,22 \mathrm{~b}$ & $160,50 \mathrm{~d}$ & $19,30 \mathrm{a}$ & $65,40 \mathrm{c}$ & $3,00 \mathrm{a}$ & -- \\
\hline M 5 M P 1 & $1,18 \mathrm{~b}$ & $369,00 \mathrm{~b}$ & $18,34 \mathrm{a}$ & $70,68 \mathrm{~b}$ & $3,00 \mathrm{a}$ & $5,22 \mathrm{~d}$ \\
\hline M 3 M P 6 & $1,17 \mathrm{~b}$ & $470,00 \mathrm{~b}$ & $14,26 \mathrm{~b}$ & $70,64 \mathrm{~b}$ & $2,00 \mathrm{~b}$ & $11,35 \mathrm{~d}$ \\
\hline M 1 PP 2 & $1,14 \mathrm{~b}$ & $656,00 \mathrm{a}$ & $16,85 \mathrm{a}$ & $64,36 \mathrm{c}$ & $2,50 \mathrm{a}$ & $7,67 \mathrm{~d}$ \\
\hline М 6РP 2 & $1,14 \mathrm{~b}$ & $461,00 \mathrm{~b}$ & $13,08 \mathrm{~b}$ & $64,74 \mathrm{c}$ & $1,00 \mathrm{~b}$ & $11,54 \mathrm{~d}$ \\
\hline M 8 M P 3 & $1,13 \mathrm{~b}$ & $365,00 \mathrm{~b}$ & $19,18 \mathrm{a}$ & $65,75 \mathrm{c}$ & $2,00 \mathrm{~b}$ & - \\
\hline M 4 M P 3 & $1,12 \mathrm{~b}$ & $258,00 \mathrm{c}$ & $20,45 \mathrm{a}$ & $62,80 \mathrm{c}$ & $2,75 \mathrm{a}$ & $5,10 \mathrm{~d}$ \\
\hline M 4PP 2 & $1,11 \mathrm{~b}$ & $294,11 \mathrm{c}$ & $16,22 \mathrm{~b}$ & $68,05 \mathrm{~b}$ & $2,28 \mathrm{~b}$ & $14,18 \mathrm{c}$ \\
\hline M 1 M P 7 & $1,08 \mathrm{~b}$ & $272,50 \mathrm{c}$ & $14,79 \mathrm{~b}$ & $68,78 \mathrm{~b}$ & $2,50 \mathrm{a}$ & $16,43 \mathrm{c}$ \\
\hline М 2 PР 5 & $1,07 \mathrm{~b}$ & $336,40 \mathrm{c}$ & $20,81 \mathrm{a}$ & $67,79 \mathrm{~b}$ & $2,00 \mathrm{~b}$ & $14,10 \mathrm{c}$ \\
\hline M 4 M P 8 & $1,07 \mathrm{~b}$ & $317,50 \mathrm{c}$ & $14,81 \mathrm{~b}$ & $70,13 \mathrm{~b}$ & $2,50 \mathrm{a}$ & $75,30 \mathrm{a}$ \\
\hline M 1 M P 3 & $1,05 \mathrm{~b}$ & $104,00 \mathrm{~d}$ & $6,05 \mathrm{~d}$ & $80,58 \mathrm{a}$ & -- & - \\
\hline M9M P3 & $1,03 \mathrm{~b}$ & $227,00 \mathrm{c}$ & $14,17 \mathrm{~b}$ & $63,58 \mathrm{c}$ & $2,50 \mathrm{a}$ & $4,65 \mathrm{~d}$ \\
\hline M 2 M P 3 & $0,92 \mathrm{~b}$ & $146,20 \mathrm{~d}$ & $4,87 \mathrm{~d}$ & $79,58 \mathrm{a}$ & -- & -- \\
\hline M 2 M P 8 & $0,82 \mathrm{~b}$ & $259,00 \mathrm{c}$ & $16,22 \mathrm{~b}$ & $67,95 \mathrm{~b}$ & $2,00 \mathrm{~b}$ & $6,30 \mathrm{~d}$ \\
\hline Média & 1,22 & 320,07 & 14,62 & 70,81 & 2,61 & 1,03 \\
\hline C.V. $(\%)$ & 8,07 & 26,05 & 21,69 & 4,53 & 42,14 & 52,47 \\
\hline
\end{tabular}

${ }^{1}$ Médias seguidas da mesma letra, na coluna, não diferem estatisticamente, pelo teste Scott-Knott, a 5\%.

${ }^{2} \mathrm{PMF}=$ Peso médio de fruto; $\mathrm{CF}=$ Comprimento de fruto; LF = Largura de fruto; ECASC = Espessura de casca; \% POLP = Percentagem de polpa; \% CASC

$=$ Percentagem de casca; $\%$ SEM $=$ Percentagem de sementes; NSEM/F = Número de sementes/fruto; NSP/F = Número de secção partenocárpica/fruto;

STT $=$ Teor de sólidos solúveis totais; ATT = Acidez total titulável.

* Bacuri sem sementes.

TABELA 5 - Correlações fenotípicas $\left(\mathrm{r}_{\mathrm{p}}\right)$ entre pares de características físicas e químicas de frutos de bacuri coletados de matrizes localizadas no Meio-Norte do Brasil.

\begin{tabular}{cccc}
\hline Pares de Características & $\mathrm{r}_{\mathrm{p}}$ & Pares de Características & $\mathrm{r}_{\mathrm{p}}$ \\
\hline P M F - P M P & $0,91^{* *}$ & E C A S C - \% C A S C & $0,99^{* *}$ \\
P M F - L F & $0,88^{* *}$ & E C A S C - \% P O L P & $0,82 * *$ \\
P M P - L F & $0,87 * *$ & P M F - C F & $0,85 * *$ \\
C F - E C A S C & $0,89 * *$ & N S E M / F - L F & $-0,75^{* *}$ \\
C F - \% C A S C & $0,89 * *$ & N S E M /F - N S P F & $-0,68 * *$
\end{tabular}

${ }^{1} \mathrm{PMF}=$ Peso médio de fruto; $\mathrm{PMP}=$ Peso médio de polpa; $\mathrm{CF}=$ Comprimento de fruto; $\mathrm{LF}=$ Largura de fruto; ECASC $=$ Espessura de casca; $\%$ CASC $=$ Percentagem de casca; \% POLP = Percentagem de polpa, NSEM/F = Número de sementes/fruto; NSP/F = Número de secção partenocárpica/fruto.

** Significativo a 0,01 . 
TABELA 6 - Estimativas de repetibilidade (r) de 14 características físicas e químicas de frutos de bacuri coletados no Meio-Norte do Brasil.

\begin{tabular}{cc}
\hline C aracterísticas & r \\
\hline C F & 0,96 \\
L F & 0,93 \\
Re la ça C F / F & 0,91 \\
P M F & 0,92 \\
P M P & 0,61 \\
\% C A S C & 0,96 \\
\% P O L P & 0,50 \\
\% S E M & 0,78 \\
E C A S C & 0,92 \\
N S E M / F & 0,71 \\
N S P / F & 0,62 \\
S S T & 0,92 \\
A T T & 0,98 \\
R e la çã o S T T / A T T & 0,93 \\
\hline
\end{tabular}

$1 \mathrm{CF}=$ Comprimento de fruto; $\mathrm{LF}=$ Largura de fruto; PMF = Peso médio de fruto; PMP = Peso médio de polpa; \% CASC = Percentagem de casca; \% POLP $=$ Percentagem de polpa; $\%$ SEM $=$ Percentagem de semente; ECASC $=$ Espessura de casca; NSEM/F = Número de sementes/fruto; NG/F = Número de gomos/ fruto; SST = Teor de sólidos solúveis totais; e ATT = Acidez total titulável.

As médias obtidas para a relação STT/ATT indicam alta variabilidade das matrizes para essa característica, com variação de 4,65 a 75,30, superior à obtida por Guimarães et al. (1992). A matriz $\mathrm{M}_{4} \mathrm{MP}_{8}$ (Brejo) apresentou a melhor performance para essa relação, seguida da matriz $\mathrm{M}_{4} \mathrm{MP}_{9}$ (Santa Quitéria). Cerca de $52,38 \%$ das matrizes avaliadas para essa característica tiveram valores inferiores a 12,0. A média de todas as matrizes ficou em 16,66 , sendo superior aos valores obtidos por Barbosa et al. (1979), Santos et al. (1988) e Nazaré (2000), e inferior aos valores médios obtidos por Guimarães et al. (1992) e Teixeira (2000). Devese salientar, contudo, que a relação SST/ATT depende, além do germoplasma em si, do estágio de maturação do fruto. Portanto, boa parte das diferenças encontradas para essa característica, em diferentes estudos, devem ser devido a esse fator.

Altos valores de correlações fenotípicas $\left(r_{p} \geq 0,85\right)$ foram obtidos para: PMF e PMP; ECASC e \% CASC; PMF e LF; PMP e CF; PMP e LF; CF e ECASC, e CF e \% CASC (Tabela 5), indicando que é possível, por exemplo, aumentar o teor de polpa do fruto através da seleção indireta para frutos mais arredondados ou para frutos mais pesados. Correlações negativas e relativamente elevadas foram obtidas para NSEM/F e LF $\left(r_{p}=-0,75\right)$ e NSEM/F e NSP/F $\left(r_{p}=-0,68\right)$, indicando que frutos mais arredondados tendem a apresentar menos sementes, e frutos com maior número de sementes tendem a produzir menos secções partenocárpicas. Em geral, características importantes como PMF, \% POLP, \% CASC, NSP/F e relação STT/ATT não se mostraram fenotipicamente correlacionadas, não significando, porém, que essas não estejam geneticamente correlacionadas (Hill \& Leath, 1975).

As estimativas de repetibilidade variaram de 0,50 (\% POLP) a 0,98 (ATT) (Tabela 6), indicando ampla variabilidade das características físicas e químicas de frutos do bacurizeiro em relação ao efeito do ambiente permanente. A ausência de efeitos gênicos outros que não o devido aos efeitos aditivos dos genes influenciando determinada característica, é importante, pois indica que simples procedimentos de seleção podem ser utilizados para melhorar a característica em consideração (Souza et al., 1998).

\section{CONCLUSÕES}

1. O germoplasma coletado no Piauí mostrou ser mais promissor em termos de potencial de uso que o coletado no Maranhão, principalmente em relação ao tamanho de fruto, peso médio e percentagem de polpa.

2. Em Caxias, no Maranhão, e em Palmeirais e Barras, no Piauí, encontraram-se as matrizes mais promissoras, especialmente em termos de peso médio de fruto e percentagem de polpa, e com maiores possibilidades de uso imediato em cultivos comerciais de bacurizeiro.

3. As altas estimativas de repetibilidade e de correlações fenotípicas obtidas para algumas características e combinações destas, respectivamente, são um indicativo de que a ampla variabilidade fenotípica observada nesse germoplasma pode ter um forte componente genético.

\section{REFERÊNCIAS BIBLIOGRÁFICAS}

ASSOCIATION of Official Analytical Chemists. 12.ed. Washington, D.C., 1975. 1015p.

BARBOSA, W.C.; NAZARÉ, R.F.R. de; NAGATA, I. Estudos físicos e químicos dos frutos: bacuri (Platonia insignis), cupuaçu (Theobroma granndiflorum e muruci (Byrsanima crassifolia). In: CONGRESSO BRASILEIRO DE FRUTICULLTURA, 5., 1979, Pelotas, RS. Anais... Pelotas: SBF, 1979. V.2, p.797-808. 
CARVALHO, J.E. U. de; NASCIMENTO, W.M.O. de; MÜLLER, C.H. Sistemas alternativos para a formação de mudas de bacurizeiro (Platonia insignis Mart.). Belém: Embrapa Amazônia Oriental, 1999. 18p. (Embrapa Amazônia Oriental. Comunicado Técnico, 11).

CAVALCANTE, P.B. Frutas comestíveis da Amazônia. 6.ed. Belém: CNPq/Museu Paraense Emílio Goeldi, 1996. 279p.

FERREIRA, F.R.; FERREIRA, S.A. do N.; CARVALHO, J.D.U. de. Espécies frutíferas pouco exploradas, com potencial econômico e social para o Brasil. Rev. Bras. Frutic., v.9, p.11-22, 1987.

GUIMARÃES, A. D. G. Coleta de germoplasma de bacuri (Platonia insignis Mart.) na Amazônia. I. Microrregião Campos do Marajó (Soure/Salvaterra). Belém: EMBRAPA-CPATU, 1992. 23p. (EMBRAPA-CPATU. Boletim de Pesquisa, 132).

Hill, R.R, Jr., and K.T. Leath. Genotypic and phenotypic correlations for reaction to five foliar pathogens in alfalfa. Theor. Appl. Genet., v.45, p.254-258, 1975.

LITTELL, R.C.; FREUND, R.J.; SPECTOR, P.C. SAS for linear models. 3.ed. Cary: SAS institute Inc., 1994. 329p.

MACEDO, M. Contribuição ao estudo de plantas econômicas no Estado do Mato Grosso. Cuiabá: UFMT, 1995. 70p.

MORAES, V.H. de F.; MÜLLER, C.H.; SOUZA, A.G.C. de;
ANTÔNIO, I.C. Native fruit species of economic potential from the brazilian Amazon. Angewandte Botanik. v.68, p.47-52, 1994.

NAZARÉ, R.F.R. de. Produtos agroindustriais de bacuri, cupuaçu, graviola e açaí, desenvolvidos pela Embrapa Amazônia Oriental. Belém: Embrapa Amazônia Oriental, 2000. 27p. (Embrapa Amazônia Oriental. Documentos, 41).

SANTOS, M. do S. S.A.; CRIÁ, F.H.; GUEDES, Z.B. de L.; BARROSO, M.A.T.; HOLANDA, L.F.F. de. Caracterização física e química de bacuri (Platonia insignis Mart.) e processamento de néctares. Boletim do Centro de Pesquisa e Processamento de Alimentos, v.6, n.2, p.73-78, 1988.

SOUZA, V.A.B. de; BYRNE, D.H.; TAYLOR, J.F. Heritability, genetic and phenotypic correlations, and predicted response to selection of several quantitative fruit traits in peach. Journal of the American Society for Horticultural Science, v.123, n.4, p.604-611, 1998b.

TEIXEIRA, G.H. de A. Frutos do bacurizeiro (Platonia insignis Mart.): caracterização, qualidade e conservação. Jaboticabal: UNESP-FCAV, 2000. 106p. (Tese de Mestrado).

VILLACHICA, H.; CARVALHO, J.E.U. de; MÜLLER, C.H.; DIAZ, S.C.; ALMANZA, M. Frutales y hortaliças promossoras de la Amazônia. Lima: Tratado de Cooperación Amazonica. Secretaria Pro-Tempore, 1996. p.152-156(TCA-SPT. Publicaciones, 44). 